\title{
Velocity fluctuations and dispersion in a simple porous medium
}

\author{
Fabrizio Capuani, ${ }^{1, *}$ Daan Frenkel, ${ }^{1, \dagger}$ and Christopher P. Lowe ${ }^{2}$ \\ ${ }^{1}$ FOM Institute for Atomic and Molecular Physics, Kruislaan 407, 1098 SJ Amsterdam, The Netherlands \\ ${ }^{2}$ Department of Chemical Engineering, University of Amsterdam, Nieuwe Achtergracht 166, 1018 WV Amsterdam, The Netherlands
}

(Received 6 November 2002; published 19 May 2003)

\begin{abstract}
We model a fluid-filled disordered porous medium by a lattice-Boltzmann system with randomly broken links. The broken links exert a friction on the fluid without excluding volume. Such a model closely mimics the idealized picture of a porous medium, which is often used in the theoretical analysis of hydrodynamic dispersion. We find that the Brinkman equation describes both the mean flow characteristics and the spatial decay of velocity fluctuations in the system. However, the temporal decay of the velocity correlations (that a particle experiences as it moves with the fluid), cannot be simply related to the spatial decay. It is this temporal decay that determines the dispersivity. Thus, hydrodynamic dispersion is generally greater than theories based on spatial correlations would imply. This is particularly true at high densities, where such theories considerably underestimate both the magnitude and transient time scale for dispersion. Nonetheless, temporal velocity correlations are still ultimately screened and the hydrodynamic dispersion coefficient converges exponentially. The long-lived transients reported for more realistic systems must therefore be due explicitly to the presence of excluded volume.
\end{abstract}

DOI: 10.1103/PhysRevE.67.056306

PACS number(s): 47.55.Mh, 05.60.-k, 46.65.+g

\section{INTRODUCTION}

When a tracer particle is introduced into a stationary fluid, it will be dispersed by Brownian motion. The dispersion can be characterized by the mean of the squared displacement in a given direction, $\Delta x^{2}$. From the Einstein definition of the self-diffusion coefficient $D_{0}$, this increases linearly with time, the constant of proportionality being twice $D_{0}$,

$$
\left\langle\Delta x^{2}(t)\right\rangle=2 D_{0} t .
$$

For a stationary fluid filling the voids in a (nonadsorbing) porous medium, the motion of the tracer particles is hindered by the medium and the diffusion coefficient of the tracer particles is reduced relative to $D_{0}$. If, on the other hand, the fluid flows through the porous medium with a mean velocity $\bar{V}$, then the dispersion of tracer particles (now defined by the variance in their displacements) increases and can become very large compared to $2 D_{0} t$. The origin of this "hydrodynamic" dispersion lies in the fact that, even in the absence of Brownian motion, different particles experience different local flow velocities and are, therefore, transported by convection over different distances in a given time $t$. Quantitatively, the dispersion coefficient is related to the time integral of the time correlation function of the velocity fluctuations experienced by tracer particles:

$$
D=\int_{0}^{\infty}\left\langle\left[v_{i}(0)-\bar{V}\right]\left[v_{i}(t)-\bar{V}\right]\right\rangle d t,
$$

where $v_{i}(t)$ is the instantaneous velocity of a particle along the flow direction as it moves through the fluid. Note that we follow convention here and refer to the diffusion coefficient

\footnotetext{
*Electronic address: capuani@amolf.nl

${ }^{\dagger}$ Electronic address: frenkel@amolf.nl
}

for the equilibrium case (where there is no flow), and to the dispersion coefficient for the nonequilibrium case (where the fluid flows). The relative importance of this convective dispersion, relative to simple diffusion, can be characterized by the Peclet number Pe. It is defined as $\mathrm{Pe}=U^{*} l^{*} / D_{0}$, where $U^{*}$ is a characteristic velocity and $l^{*}$ is a characteristic length. The obvious choice for the characteristic velocity $U^{*}$ is the mean velocity of the fluid $\bar{V}$. At high Peclet numbers, tracer transport over distances larger than $l^{*}$ is dominated by convection, and dispersion is therefore dominated by the spatial fluctuations in fluid velocity. Conversely, at low Peclet numbers, the convective contribution is small and simple diffusion dominates

In order to understand hydrodynamic dispersion, we need an idea of how fluid flows in porous media. If the fluid is Newtonian, then the steady-state velocity fields $\mathbf{v}$ will be solutions of the time-independent Navier-Stokes equations

$$
\begin{gathered}
-\boldsymbol{\nabla} P+\eta \nabla^{2} \mathbf{v}+\mathbf{F}=0, \\
\boldsymbol{\nabla} \cdot \mathbf{v}=0
\end{gathered}
$$

that satisfy stick boundary conditions at the solid/fluid interface. Here $P$ is pressure, $F$ is any external forces acting on the fluid, and $\eta$ is the viscosity. We have also assumed that inertia is negligible (the flow is at low Reynolds number). The relation between the steady-state flow velocity and the applied pressure gradient is then given by the empirical Darcy's law,

$$
\bar{V}=-\frac{\kappa}{\eta} \nabla P,
$$

where $\kappa$ is a constant (the permeability) that depends only on the properties of the porous medium, not on those of the fluid. Equation 4 is a first-order equation while Eqs. (3) are second-order equations. It is therefore impossible to formu- 
late rational boundary conditions between the two. In 1947 Brinkman proposed an equation to describe the locally averaged flow in a porous media [1]. The Brinkman equation considers the balance of forces acting on a volume element of fluid, i.e., the pressure gradient, the divergence of the viscous stress tensor, and the friction force exerted by the porous medium:

$$
\eta \nabla^{2} v-\nabla P-\frac{\eta}{\kappa} v=0
$$

The crucial assumption is that the external force in the Navier-Stokes equation [Eq. (3)] can be replaced by the force term in Darcy's law [Eq. (4)]. This substitution is only justified if the porous medium occupies a vanishingly small fraction of space. In that case one may consider the porous medium as a continuum that exerts a friction on the fluid at every point. In spite of the approximate nature of the Brinkman equation, it has proved to be an extremely useful tool for modeling flow in spatially inhomogeneous porous media $[2,3]$.

Of most relevance to us here is the use of the Brinkman equation to describe not average flow velocities, but the spatial decay of fluctuations in the flow velocity. It is clear from Eq. (2) that it is these fluctuations that play the crucial role in determining the dispersion coefficient. Indeed, if Brownian motion can be neglected, the particle velocity $v_{i}(t)$ appearing in Eq. (2) is simply the instantaneous velocity of a particle as it convects along a streamline. This we refer to as the Lagrangian velocity correlation function $C_{v}(t)$. This concept was utilized by Koch and Brady in their theoretical analysis of dispersion in random media composed of randomly distributed fixed particles. Notably, they made use of the fact that a velocity fluctuation generated by one of the fixed points making up the porous medium will, according to the Brinkman theory, decay in space on a length scale set by the Brinkman length $\lambda$. The Brinkman length is the square root of the permeability. If the particles making up the medium have no spatial extension (they are simply points in the fluid exerting friction), the decay is exponential. If they do have a spatial extension, in the sense that stick boundary conditions apply on the surface, the decay is slower, going with distance $r$ as $1 / r^{3}$ [4]. On the other hand, if the presence of the porous medium is neglected, the Brinkman equation reduces to the usual Navier-Stokes equation for which a velocity perturbation decays as $1 / r$. This leads to an unbounded integral for the dispersion coefficient, implying that the dispersion coefficient diverges, that is, it would always depend on the system size. The hydrodynamic screening predicted by the Brinkman equation thus plays a crucial role in determining the dispersion coefficient. A similar effect occurs in sedimentation, where velocity fluctuations in an unbounded system diverge [5]. In this case, it is the presence of container walls that is crucial in providing the necessary screening [6].

The question we want to address here is how well this picture, central to the theory of Koch and Brady, describes hydrodynamic dispersion. One reason for doing so is that numerical simulations of dispersion in packed beds of spheres, reported in Ref. [7], suggested that the dispersion coefficient was still increasing on time scales where the theory suggested it should have already converged. This raised the question: Is this the asymptotic behavior? For realistic packed beds of spheres Koch et al. [8] showed that the screening picture describes the decay of the velocity fluctuations reasonably well. Dorlosfky and Brady [9] arrived at the same conclusion. There are nonetheless two complications with "realistic" systems. First, as noted above, the Brinkman screening is less dramatic (going from a $1 / r$ decay to $1 / r^{3}$ ) for spatially extended particles than is the case for points (from $1 / r$, to exponential). Second, the presence of an explicit solid/fluid interface, where the flow velocity goes to zero, means that there is a region close to the surface that the tracer particles must always enter and leave by diffusion. Koch and Brady suggest that the presence of this diffusive boundary layer means that the dispersion coefficient reaches its asymptotic value on time scales much longer than would otherwise be expected. This could also be responsible for the behavior observed in Ref. [7]. In this paper we, therefore, consider a simple model system where both these complications are absent; that is, following in the spirit of the theory, we consider a porous medium composed of fixed points that exert friction but have no spatial extension. Indeed, the analysis of such a system gives one contribution to the overall dispersion coefficient in the expression derived for a packed bed [10]. It is regarded as the contribution to the dispersion coefficient due to the velocity perturbation at distances far from the fixed particle. Specifically, this purely convective term, $D_{c}$, makes a contribution to the total dispersion coefficient

$$
D_{c}=\frac{\bar{V}}{8 \pi \rho \lambda^{2}},
$$

where $\rho$ is the number density of the fixed points. For the work we describe here, we will ignore Brownian motion and concentrate solely on the decay of velocity fluctuations due to convection. In that case, we have $D=D_{c}$. While the model may seem of somewhat academic interest, there are important examples of hydrodynamic dispersion in dilute systems for which the model could be reasonably applied. Dispersion in flow through polymer networks would be an example.

\section{DESCRIPTION OF THE MODEL}

To simulate fluid flow in our model porous media, we employed the lattice-Boltzmann (LB) method. This method describes the fluid in terms of the density of particles with a discrete set of velocities, constraining their locations to a discrete set of positions $\mathbf{r}$ at integer times. These positions thus correspond to points on a lattice when time is discretized. The choice of the lattice is restricted by the fact that only a few lattices have a high enough local symmetry to model hydrodynamic flow with an isotropic viscosity. The choice of the lattice defines the velocities $\left\{\mathbf{c}_{i}\right\}$ that are allowed. In our simulations we have used the "D3Q18" model [11], where D3 indicates that the lattice is three dimensional and the number after the "Q" indicates the number of veloc- 
ity allowed per site (in our case 18) [12]. For more details on possible lattices see, for example, Ref. [12]. The quantity we calculate in the LB method is the discretized one-particle velocity distribution function $n_{i}(\mathbf{r}, t)$. This is the probability a particle at lattice site $\mathbf{r}$ at time $t$ has a velocity $\mathbf{c}_{i}$. The hydrodynamic fields, the density $\rho(\mathbf{r})$, and momentum density $\mathbf{j}(\mathbf{r})$, for example, are moments of this distribution function,

$$
\begin{aligned}
& \rho(\mathbf{r}, t)=\sum_{i} n_{i}(\mathbf{r}, t) ; \\
& \mathbf{j}(\mathbf{r}, t)=\sum_{i} \mathbf{c}_{i} n_{i}(\mathbf{r}, t),
\end{aligned}
$$

where $i$ sums over all possible velocities $\mathbf{c}_{i}$. The time evolution of the distribution function is described by the discretized analog of the Boltzmann equation [11].

$$
n_{i}\left(\mathbf{r}+\mathbf{c}_{i}, t+1\right)=n_{i}(\mathbf{r}, t)+\Delta_{i}(\mathbf{r}, t) .
$$

Here, $\Delta_{i}$ is the change in $n_{i}$ due to "collisions" at the lattice sites. The postcollision distribution $n_{i}+\Delta_{i}$ is propagated in the direction of the velocity vector $\mathbf{c}_{i}$. A complete description of the collision process is given in Ref. [11]. The main effect of the collision operator $\Delta_{i}(\mathbf{r}, t)$ is to (partially) relax the shear stress at every lattice site while conserving the local particle number and momentum. The rate of stress relaxation is related to the kinematic viscosity $\nu$. A full description of the collision operator is given by Ladd [11]. Further, we make use of the simplest collision operator where the nonequilibrium components of the stress tensor relax in one time step and the Reynolds number is rigorously zero (the convective term in the equilibrium stress tensor is neglected). The overall procedure involves two steps: a propagation step and a collision step. In the propagation step each distribution function is moved to the neighboring site by velocity $\mathbf{c}_{i}$. In the collision step the distributions at each site "collide," in the sense that they are modified by the collision operator. It can be shown that the evolution of the hydrodynamic fields in the model is described by the Navier-Stokes equations [11].

Having described how we can simulate the fluid, we now need a method to model the porous medium. Within the lattice-Boltzmann framework there is a straightforward procedure for imposing stick boundary conditions at an explicit solid/fluid interface. A simple bounce-back rule performed on boundary links enforces the stick boundary condition to second order, while not perturbing the stress [11]. Boundary links are defined as links connecting lattice sites inside and outside the solid object, and obviously these come in pairs. Adopting a convention of labeling the link that goes from inside to outside as $i b$ and its partner $-i b$,

$$
\begin{gathered}
n_{-i b}\left(\mathbf{r}_{b}, t+\Delta\right)=n_{i b}(\mathbf{r}, t), \\
n_{i b}\left(\mathbf{r}_{b}+\mathbf{c}_{i b}, t+\Delta\right)=n_{-i b}\left(\mathbf{r}_{b}+\mathbf{c}_{i b}, t\right),
\end{gathered}
$$

Here we do not want an explicit solid-fluid interface, in the sense of a solid phase that excludes volume from the fluid.
Instead, with a given probability, we break links [that is, define a set of links for which the propagation equation is modified according to Eq. (10)]. These broken links exclude no volume (so long as the fraction of broken links stays well below the percolation threshold) but will exert a friction on the fluid proportional to the local flow velocity. They are effectively point scatterers.

\section{RESULTS}

To generate our model porous medium we generated a set of point sources for friction according to a binomial distribution. We first fixed the probability $\Phi$ that a link would be broken. This number was varied between 0.01 and 0.3 . For every link, we then generated a random number between 0 and 1 . If this random number was less than $\Phi$, the link was broken, otherwise it was left intact. In this way, we generated one particular realization of the porous medium for a given value of $\Phi$. All our results were obtained by performing simulations for at least 25 different configurations for every value of $\Phi$. Note that, for every configuration, the fraction of broken links is not exactly equal to $\Phi$. Only the average value is imposed. We cannot predict a priori how the Brinkman length depends on the fraction $\Phi$ of broken links, because this, even for point scatters, involves the many-particle hydrodynamic interactions. We therefore determine $\lambda$ numerically. This can be done in two ways. One is to measure the average flow velocity in the presence of an applied external force and calculate the permeability. The second is to compute the flow profile in a simple confined geometry. For instance, the Brinkman equation (5) can easily be solved for a three-dimensional porous medium confined in a slit bounded by two hard walls. On the walls, stick boundary conditions apply. If we apply a body force parallel to the plates, the solution for the steady velocity profile is given by

$$
v_{x}(z)=\frac{\lambda^{2} F}{\eta}\left[1-\frac{\cosh \left(\frac{z}{\lambda}\right)}{\cosh \left(\frac{L}{2 \lambda}\right)}\right],
$$

where the two plates are located at $z=-L / 2$ and $z=+L / 2, x$ is the direction of the force and $F$ is a force per unit of volume. Note that the Brinkman length $\lambda$ enters this equation twice: first through the (Darcy) prefactor, and second through the "screening" length that determines the shape of the flow profile. The constraint that a single value of $\lambda$ should fit both the shape and the prefactor provides a good consistency check on our determination of $\lambda$.

The simulation box had a length of 320 lattice spacings in the direction of the flow, and 40 lattice spacings in the other two directions. Periodic boundary conditions were used in the unbounded directions. Even for the lowest density system $(\Phi=0.01)$, the Brinkman length was found to be no larger than 3.41 lattice units. This is more than an order of magnitude less than the smallest system dimension. At larger values of $\Phi$, the Brinkman length is even smaller. Hence, we expect finite-size effects to be negligible at all values of $\Phi$ that we studied. To study the flow, we let the system evolve 


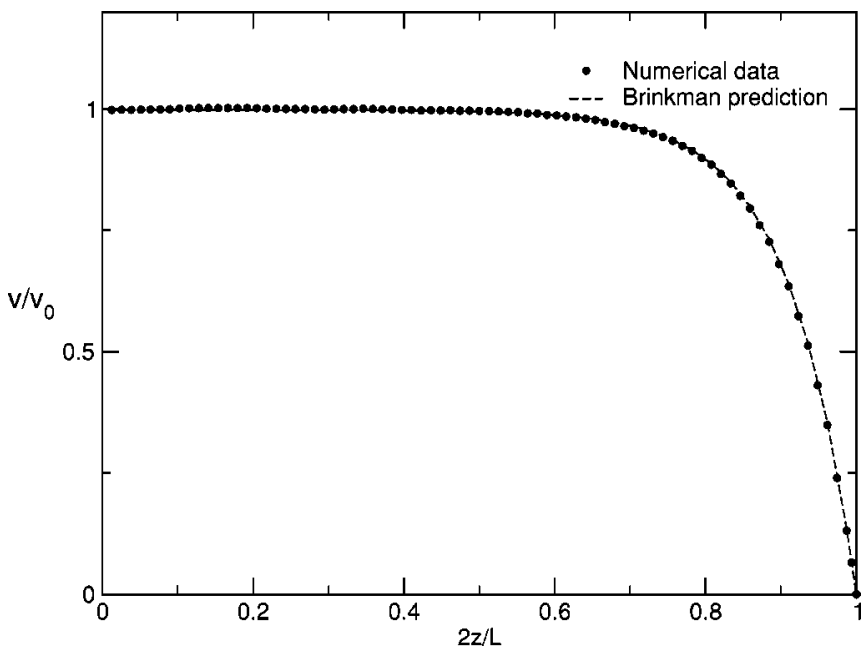

FIG. 1. Velocity profile for a fluid flowing through the model porous medium confined between two plates. The flow velocity $v$ normalized by the maximum flow velocity $v_{0}$ is plotted as a function of dimensionless distance from the center. The dashed line is the solution of the Brinkman equation; the circles are the numerical data.

under the applied body force, $F$. After some transient time, the flow fields reach a steady state. All correlation functions that we report have been computed for this steady state. To compute the average flow profile, we averaged the steadystate flow profiles of all 25 different configurations. It is to this averaged flow profile that we fitted the Brinkman flow profile given by equation (11). A result of this fitting procedure is shown in Fig. 1. It is worth remembering that the Brinkman length is the only parameter in this fit. The figure shows that the computed flow profile fits the Brinkman expression. This result is not a priori obvious and provides a useful check that we do indeed have a system with a welldefined Brinkman length whose spatially averaged behavior is a solution to the Brinkman equation. By repeating this fitting procedure for other values of $\Phi$, we obtained the dependence of $\lambda$ on $\Phi$. We found that the low density result $\lambda \propto 1 / \sqrt{\Phi}$ holds to a good approximation over the whole range of $\Phi$ we consider here.

As Fig. 1 shows, the Brinkman length describes the distance over which a flow profile in a porous medium is perturbed by an "obstacle" (in this case the hard wall). In the spirit (if not the letter) of Onsager's regression hypothesis, we might expect that "spontaneous" spatial velocity fluctuations should decay on the same length scale. To verify this, we computed the spatial velocity correlation function (SVCF) defined as

$$
C_{s}(r)=\langle[v(0)-\bar{V}][v(r)-\bar{V}]\rangle,
$$

where $v(r)$ is the component of fluid velocity along the flow direction at a distance $r$. To compute the SVCF (and all remaining correlation functions), we considered a purely periodic system without walls. In such a system, the average flow velocity is the same everywhere. In any specific realization of the disorder, however, there will be local deviations

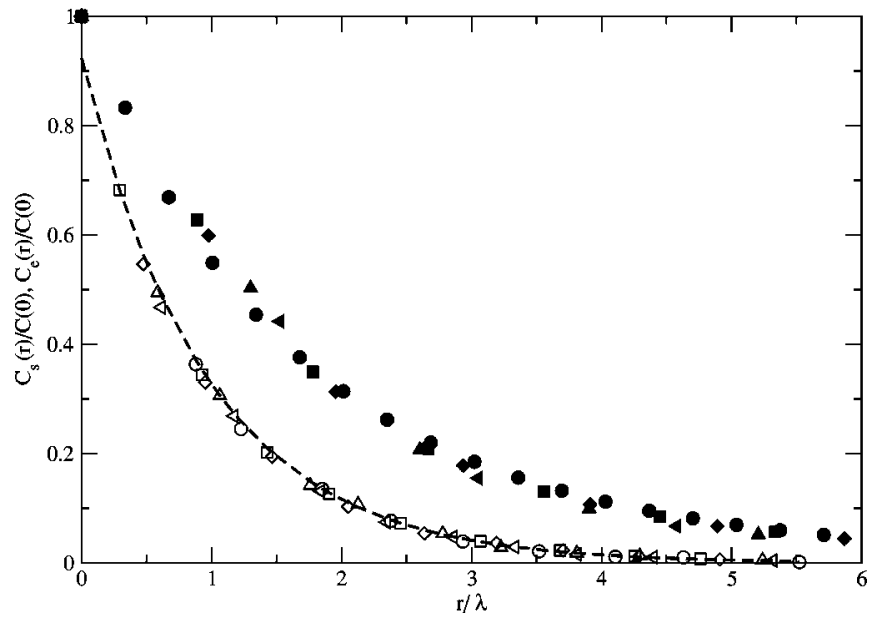

FIG. 2. The normalized spatial velocity correlation function $\left[C_{s}(r) / C(0)\right.$, open symbols] and Eulerian velocity correlation function $\left[C_{e}(r) / C(0)\right.$, filled symbols], as a function of dimensionless distance $r / \lambda$. The broken link fractions are $\Phi=0.01$ (plus), 0.08 (circles), 0.1 (squares), 0.2 (diamonds), and 0.3 (triangles). The error bars are smaller than symbols. The dashed line is the result of fitting an exponential to $C_{s}(r) / C(0)$ for $r / \lambda>0.5$

from the average value. The SVCF shows how these disorder-induced fluctuations decay, spatially, to zero.

In Fig. 2 (open symbols) we show $C_{s}(r) / C_{s}(0)$ computed for a range of values of $\Phi$. In this figure, we have expressed all distances in units of the Brinkman length $\lambda$. If, as we assume, the Brinkman length is the only relevant length scale in the system, then all the functions should superimpose. This is precisely what we observe. Moreover, the SVCF decays exponentially, with a characteristic decay distance of a Brinkman length. It seems, therefore, that the average flow profiles and the averaged spatial decay of perturbations in the flow profile satisfy Brinkman scaling. It would seem logical to assume that, as the spatial decay of velocity fluctuations satisfies Brinkman scaling, so should the temporal decay. If this were true, then Brinkman scaling should apply to dispersion of tracer particles. In fact, it has been argued that the nature of the equations, in particular, that the Brinkman equation has no explicit time dependence, has as a consequence that the Lagrangian velocity correlation function (LVCF) should decay in the same way as the SVCF, i.e., exponentially, with a characteristic time equal to $\lambda / \bar{V}$ [10]. The LVCF is, within this approximation, simply

$$
C_{v}(t) \approx\langle[v(0)-\bar{V}][v(\mathbf{r}=\bar{V} t)-\bar{V}]\rangle=C_{e}(r=\bar{V} t) .
$$

We call this approximation to the LVCF the Eulerian time velocity correlation function $(\mathrm{EVCF}), C_{e}(\bar{V} t)$. In Fig. 2 (filled symbols) we show the normalized EVCF computed for a range of values of $\Phi$, together with the SVCF. We observe that all EVCF's superimpose. The typical length of decay is a little bit larger than that for the SVCF, because we are now considering correlations along the flow direction only. Otherwise, there is little difference. Based on this approximation, one would expect that the natural unit of time 


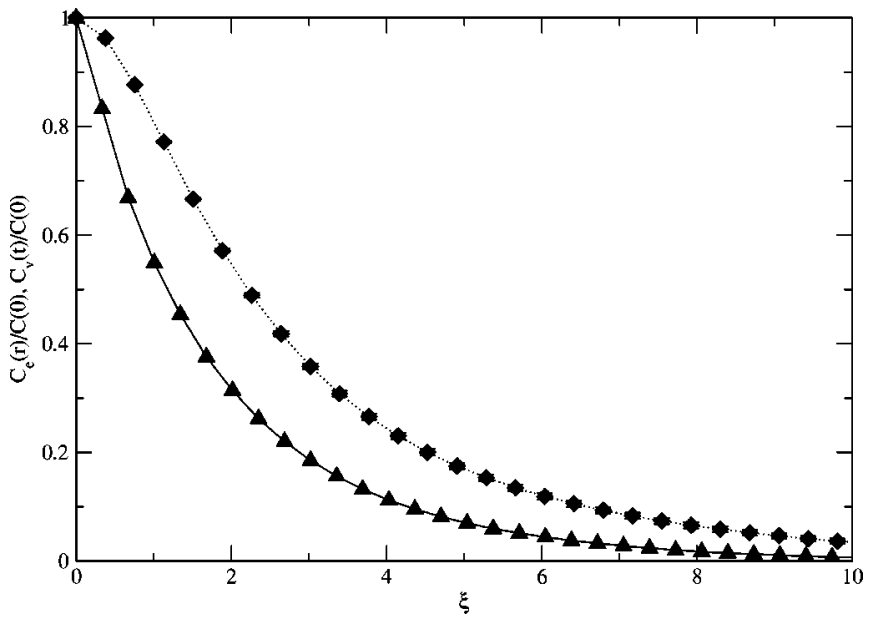

FIG. 3. Comparison of the scaled, normalized, Eulerian (triangles), and Lagrangian (diamonds) velocity correlation functions. $\xi$ is a dimensionless unit equal to $r / \lambda$ for $C_{e}(r)$ and to $t / \tau$ for $C_{v}(t)$. Time $\tau$ is the average time to convect a Brinkman length $(\tau=\lambda / \bar{V})$. The dotted lines are guides to the eye.

for the Lagrangian velocity correlation functions is the Brinkman time defined as $\tau=\lambda / \bar{V}$, i.e., the average time it takes a tracer particle to travel over a distance equal to one Brinkman length. In order to calculate the Lagrangian velocity correlation function, we need to follow the trajectory along which a particle travels; that is, we have to calculate velocity correlations for a streamline. In the appendixes we describe our procedure for doing so. Figure 3 shows the LVCF for a system with $\Phi=0.3$. In the same figure, we show the theoretical prediction for the shape of the LVCF corresponding to the EVCF and assuming that the velocity time correlation function can be obtained directly by replacing the displacement in the latter by $t \bar{V}$. The figure shows clearly that there exists no such simple relation between the spatial and temporal decays of velocity fluctuations. In the first place, there is a marked difference in the behavior at short times. The initial rate of decay of the Lagrangian function is zero, so it cannot be approximated by an exponential. It is straightforward to show, from the incompressibility condition, that this must be the case (see Appendix A). More importantly, if we plot the LVCF for different values of $\Phi$ (i.e., different Brinkman lengths), we cannot make the different LVCF's collapse onto the same master curve (see Fig. 4). This is surprising, because it suggests that the Brinkman time is not the only relevant time scale in the system. In fact, Koch and coworkers $[10,8]$ have suggested that anomalous (non-Brinkman) decay of velocity time correlation functions should be intimately linked to similar anomalies in the SVCF. Yet, our simulations appear to show "normal" behavior in the EVCF and "anomalous" behavior in the LVCF. As the LVCF decays much more slowly than one would expect on basis of the Brinkman-scaling assumption, the dispersion coefficient [calculated from Eq. (2)] is larger than would be predicted by simple use of the Brinkman equation. The slow decay of the LVCF indicates that the velocity of tracer particles remains strongly correlated in the time that it takes the average fluid to move over one Brinkman length. Hence, the

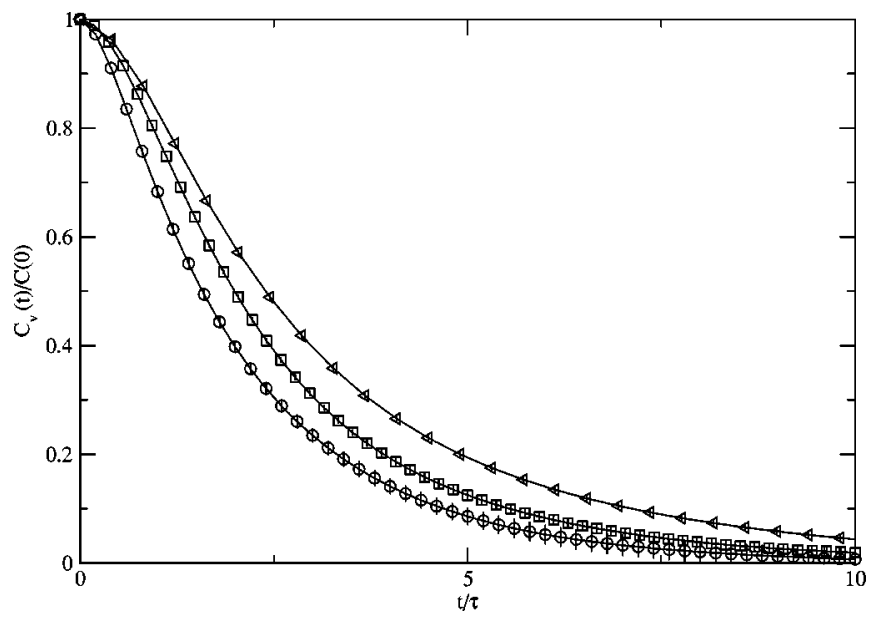

FIG. 4. The normalized Lagrangian velocity correlation function as a function of dimensionless time. The different curves correspond to different broken link fractions. The corresponding dimensionless densities are 2.4 (circles), 1.7 (squares), and 0.27 (triangles). Note that the curves do not superimpose but decay more slowly with decreasing dimensionless density (increasing absolute density).

hydrodynamic screening picture that works so well for average flow profiles, seems to be quantitatively (though not qualitatively) incorrect when we consider temporal correlations.

In what follows, we shall consider hydrodynamic dispersion in the limit of high Peclet number. In this limit, and in the absence of any explicit solid/fluid interface, the Brownian motion of the tracer particles can be ignored. It then follows from Eq. (2), that the dispersion coefficient is related to the time integral of the Lagrangian velocity correlation function. We now compare the computed dispersion coefficients with values predicted by the theory of Koch and Brady. Before proceeding, we need to briefly recapitulate their model. They model the porous medium by a continuum of points, every point exerting a friction on the fluid. This might seem an abstract concept of porous media, but it represents a simplified model of a dilute packed bed of spheres in the limit of many scatterers per Brinkman length cubed. Using such a model, they were able to compute the fluid velocity perturbation at large distances generated by particles making up the porous medium. From this they derive an expression for the dispersion coefficient. In order to perform the calculation they approximate the LVCF with the EVCF. The resulting expression for the dispersion coefficient at high Peclet number is

$$
\frac{D_{t h}}{D_{0}}=\frac{3}{4} \mathrm{Pe},
$$

where the Peclet number is $\mathrm{Pe}=U^{*} l^{*} / D_{0} ; U^{*}$ being naturally identified with the mean velocity of fluid $\bar{V}$ and $D_{0}$ being the diffusion coefficient [here superfluous because it cancels the $D_{0}$ on the left hand side of Eq. (14)]. Some discussion is needed about the choice of $l^{*}$. In general, $l^{*}$ is a typical length of the system. The theory takes as a charac- 
teristic length the "effective" radius of spherical particles, which leads to a low density random medium with the same Brinkman length. This gives the expression $D_{t h}=\frac{3}{4} \bar{V} a$. In actual, radius $a$ is a somewhat fictitious parameter obtained by implying a dilute bed of spheres to model the porous medium. The following procedure is needed to obtain $a$ as function of the Brinkman length $\lambda$. A dilute bed of spheres, with number density of scatterers $\rho$, exerts a total drag equal to $\rho$ times the Stokes drag of a single sphere. Darcy's law gives a value for the same drag in terms of the Brinkman length [Eq. (4)]. Equating the two, one gets

$$
\lambda=\sqrt{\frac{1}{6 \pi \rho a}} .
$$

Using Eq. (15) the dispersion coefficient becomes

$$
D_{t h}=\frac{\langle u\rangle}{8 \pi \rho \lambda^{2}} .
$$

We need, at this point, an expression for $\rho$ in terms of the broken links model. We should point out that, at this level of detail, it is difficult to map our model directly to the theory because we have, in effect, points with a directionally dependent friction. The theory, on the other hand, considers points that exert an isotropic friction. To match (approximately) the two, we proceed as follows. In the D3Q18 lattice there are 18 links, six of which have weight 2 [11]; in our case only two such links are oriented along the flow direction. There are also eight singly occupied links with a component in the flow direction, oriented at $45^{\circ}$ to the flow. We therefore take these to contribute $1 / 2$. The remaining links are oriented perpendicular to the flow direction, so these contribute nothing. Allowing for the fact that each link belongs to two lattice sites, the effective density of links, in lattice units, we therefore take to be $\rho=4 \Phi$. From the simulation data the dispersion coefficient can be computed from the Lagrangian velocity correlation function,

$$
D=\int_{0}^{\infty} C_{v}(t) d t=C(0) \int_{0}^{\infty} C_{v}(t) / C(0) d t
$$

The initial value of the function, $C(0)$, is simply the covariance of the velocity field. This is, in fact, true for all the correlation functions we have defined. Equation (17) defines $D$. If, instead, we approximate LVCF with EVCF, we can define

$$
D_{E u l}=C(0) \int_{0}^{\infty} C_{e}(t) / C(0) d t=C(0) \tau^{\prime}
$$

As we have already shown that the $C_{e}(t) / C(0)$ scales onto a single curve $\tau^{\prime}$ defined by Eq. (18) and representing a characteristic time, is the same for all values of the Brinkman length. The two integrals will, in general, be different, in that while $C_{v}(t)$ is related to the velocity of the particle at time $t$, $C_{e}(t)$ refers to the velocity of a particle at a position $r$ $=\bar{V} t$. The two quantities are only necessarily equal in the absence of velocity fluctuations.

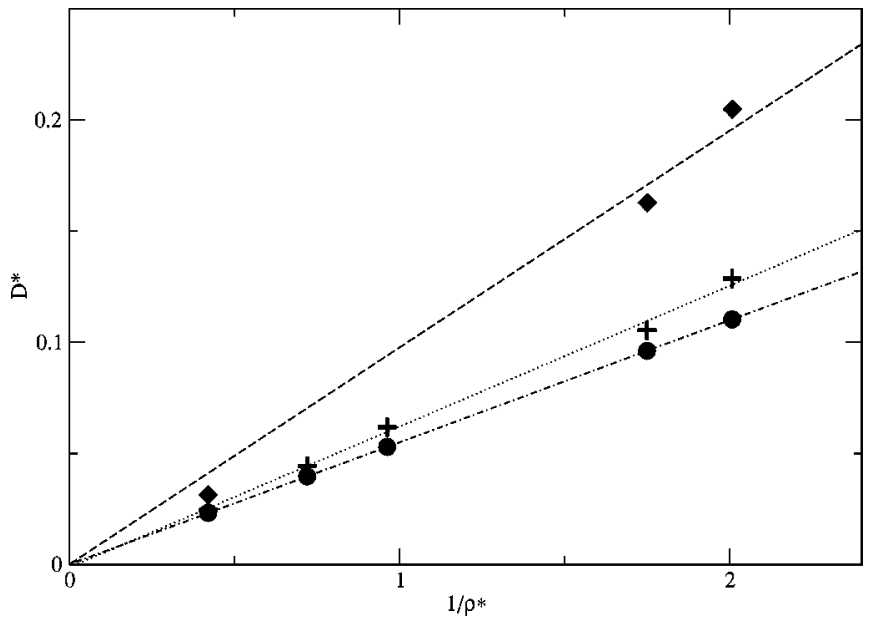

FIG. 5. The dimensionless dispersion coefficient $D^{*}$ as a function of inverse dimensionless density $\rho^{*}$. Also plotted are the theoretical values of the dimensionless dispersion coefficient $D_{t h}^{*}$ (circles) and the dispersion coefficient obtained by approximating the Lagrangian velocity correlation function with the scaled Eulerian function, $D_{E u l}^{*}$ (plus). Note that the high values of $\rho^{*}$ correspond to the low point densities so this data covers the spatially dilute regime.

In units where length, time, and velocity are, respectively, $\lambda, \tau$, and $\bar{V}$, the dispersion coefficients are thus

$$
\begin{aligned}
D_{t h}^{*} & =\frac{1}{8 \pi \rho \lambda^{3}}, \\
D_{E u l}^{*} & =\frac{C(0)}{\bar{V}^{2}} \frac{\tau^{\prime}}{\tau}, \\
D^{*} & =\frac{1}{\bar{V}^{2}} \int_{0}^{\infty} C_{v}(t) d t .
\end{aligned}
$$

In the following we will plot the dispersion coefficients as a function of the dimensionless friction point density $\rho^{*}$ $=\rho \lambda^{3}$. This is the number of scatterers per cubic Brinkman length and sets the intrinsic scale of the system. The scaling of the Brinkman length $\left[\lambda \sim \rho^{-1 / 2}\right.$, see Eq. (15)] means that dense porous media in "real word" units are actually dilute in the intrinsic scale set by the Brinkman length. In fact, when $\rho \rightarrow \infty, \rho^{*} \rightarrow 0$; that is, high dimensionless number densities correspond to the approximations made in the theory, because in the (spatially) dilute limit there are many scatterers per Brinkman length cubed. This assumption is needed to treat the porous medium as a continuum. We are thus able to disentangle the effect of the dilute limit approximation and use the EVCF instead of the LVCF to compute the dispersion coefficient.

In Fig. 5 we plot $D_{t h}^{*}$ and $D_{E u l}^{*}$ as functions of $1 / \rho^{*}$ for high values of $\rho^{*}$. The theoretical value of the dispersion coefficient $D_{t h}^{*}$ is a linear function of $1 / \rho^{*}$ [Eqs. (19)]. We observe that in the dilute limit $D_{E u l}^{*}$ also has a linear behavior. A linear fit of $D_{E u l}^{*}$ for high $\rho^{*}$ has an intercept at zero, as the theory predicts. In the figure we plotted also $D^{*}$, 


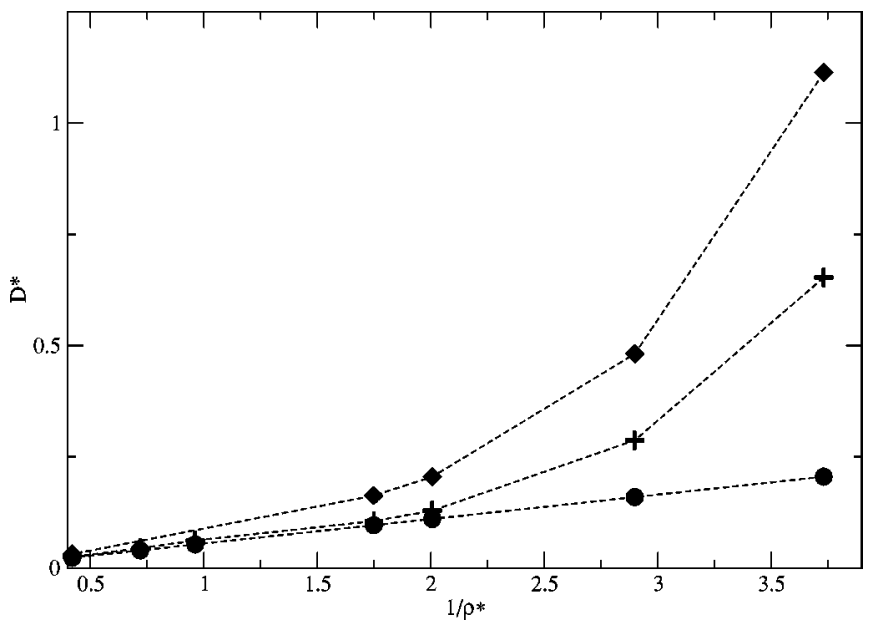

FIG. 6. The dimensionless dispersion coefficient $D^{*}$ as a function of the inverse dimensionless density $\rho^{*}$. Also plotted are the theoretical values of the dimensionless dispersion coefficient $D_{t h}^{*}$ (circles) and the dispersion coefficient obtained by approximating the Lagrangian velocity correlation function with the scaled Eulerian function $D_{E u l}^{*}$ (plus). Note that the low values of $\rho^{*}$ correspond to the high point densities so this data extends to the spatially dense regime.

which has the same behavior. At a given number density the difference between $D_{t h}^{*}$ and $D_{E u l}^{*}$ measures the effect of the dilute limit approximation, whereas the difference between $D_{E u l}^{*}$ and $D^{*}$ measures the error made by approximating the LVCF with the EVCF. The difference between $D_{t h}^{*}$ and $D^{*}$ is a measure of the accumulated effect of the two approximations. We can conclude that the theoretical expression works very well in the dilute limit. In this regime the absolute agreement between the theory and simulations, given the approximate nature of the mapping, is clearly very good. This confirms the prediction of Koch and Brady theory that the dispersivity is independent of volume fraction for dilute beds.

In Fig. 6 we show the whole range of densities covered by our simulations, and we repeat the analysis carried out for Fig. 5. Again on the abscissa is the inverse dimensionless density $1 / \rho^{*}$. For decreasing values of the dimensionless density the two lines diverge. The difference between the two represents the factor needed to correct for the dilute limit assumption. We observe that the computed dispersion coefficients no longer have a linear dependence on $1 / \rho^{*}$. We also see that there is an appreciable difference between the exact dispersion coefficient $D^{*}$ and both the approximations $D_{E u l}^{*}$ and $D_{t h}^{*}$. This difference increases with decreasing $\rho^{*}$. This means that, for what would correspond to an increasingly packed bed, the dispersion coefficient becomes much larger than the theory predicts. Both the dilute limit assumption and approximating the Lagrangian velocity correlation function with the Eulerian contribute to the error. At low and moderate densities it is the former, rather than the latter, that limits the validity of the theory.

\section{CONCLUSIONS}

In this paper we described numerical simulations of flow through a simple model porous medium. The porous medium was modeled by simply breaking randomly, with probability $\Phi$, the links used in the lattice-Boltzmann equation. From the point of view of comparing with theory, this broken link model has two advantages. First, it has no excluded volume. Second, it exerts a relatively high local friction. The latter allows one to calculate correlation functions over several Brinkman lengths. We showed, by two different calculations, that this model does indeed behave as the Brinkman equation predicts. Specifically, for the velocity profile of a flow through a porous medium sandwiched between two plates and the spatial decay of fluctuations in the local flow velocity about the mean (SVCF). The latter is an assumption in the Koch and Brady theory of dispersion in random media and it is a good one. We found that there is a universal behavior of spatial correlations if we measure lengths in units of the Brinkman length, confirming that this is the only relevant length in the system. We also confirmed that, spatially, this results in an exponential screening of the fluctuations. Again, this is central to the Koch and Brady theory. It guarantees convergence of the dispersion coefficient, which would otherwise diverge. We can conclude that the broken link model is well described by the Brinkman equation. Conversely, the Brinkman equation describes successfully the spatial decay of velocity fluctuations in our model porous medium.

From the point of view of hydrodynamic dispersion, it is the temporal, rather than spatial decay, of fluctuations that is relevant. For the Lagrangian velocity correlation function (the temporal decay of the velocity a particle experiences as it traverses the fluid) things were more complex. This "time" velocity correlation function behaved quite differently. Its decay is not a simple exponential and, if we attempt to relate time to an average displacement, there is no universal behavior in the scaled decay. The decay of this function cannot be predicted by a simple mapping to the Brinkman equation alone. It is not possible to say that Lagrangian fluctuations are uniquely related to the Eulerian fluctuations for all densities of scatterers. This is an approximation invoked in the Koch and Brady theory and one, the simulations show, that is only strictly justified for low densities (Koch and Brady themselves acknowledge that it is a low density approximation). We should stress, however, that the decay of the Lagrangian correlation function with time is still asymptotically exponential. Thus, the qualitative picture that the screening of the velocity fluctuations by the porous medium itself leads to a convergent dispersion coefficient remains true. The results reported in Ref. [7] cannot be attributed to a breakdown of the screening picture, the slow decay must in some way be related to the more complex nature of the porous medium, notably, the presence of excluded volume and diffusive boundary layers. Nonetheless, the conclusion that Lagrangian correlations can decay on much longer temporal (and, by implication, spatial) scales is consistent with Ref. [7]. Here, the correlation functions were decaying on time scales that greatly exceeded the Brinkman time.

Given the simplicity of our model and its similarity to the system Koch and Brady used to develop part of their theory of dispersion in packed beds, a direct comparison seemed appropriate. We thus computed the dispersion coefficient (in the limit where molecular diffusion can be neglected) as a 
function of broken link density. In the limit where the theory should be most valid (low volume fraction, a broken link "volume" interpreted as the volume of a sphere that exerts the same friction), we found reasonable agreement. At higher volume fractions, the agreement breaks down and the theory grossly underestimates the dispersion coefficient. In this regime, the decay of the Lagrangian velocity correlation functions differs dramatically from the scaled spatial decay used to approximate it in the theory. The fact that the theory gives dispersivities that actually agree quite well with experimental results for dense packed beds must be due to the fact that it more accurately accounts for the boundary layer dispersion that we do not consider here.

\section{ACKNOWLEDGMENTS}

We thank Marco Cosentino Lagomarsino for a critical reading of the manuscript. The work of the FOM Institute is part of the research program of FOM and was made possible by financial support from the Netherlands organization for Scientific Research (NWO). An NCF grant of computer time on the TERAS supercomputer is gratefully acknowledged.

\section{APPENDIX A: INITIAL RATE OF DECAY OF THE LVCF}

In this section we give a simple argument as to why the initial gradient of the Lagrangian velocity correlation function, computed along the direction of the flow, should be zero. We define the $x$ direction as the tangent to the trajectory $\hat{\mathbf{x}} \equiv \hat{\mathbf{t}}$. The $\hat{\mathbf{y}}$ and $\hat{\mathbf{z}}$ directions would be any pair of orthogonal vectors in the plane orthogonal to the trajectory. The LVCF is defined as $C_{x}(t)=\left\langle u_{x}(0) u_{x}(t)\right\rangle$ and its time derivative is

$$
\frac{\partial C_{x}(t)}{\partial t}=\left\langle\left. u_{x}(0) \frac{\partial u_{x}(t)}{\partial t}\right|_{t=0}\right\rangle \text {. }
$$

On the other hand, the time derivative of the fluid velocity experienced by a tracer particle can be written as

$$
\left.\frac{\partial u_{x}(t)}{\partial t}\right|_{t=0}=\left.\left.\frac{\partial x(t)}{\partial t}\right|_{t=0} \frac{\partial u_{x}(t)}{\partial x}\right|_{t=0}=\left.u_{x}(0) \frac{\partial u_{x}(t)}{\partial x}\right|_{t=0} .
$$

We can now use the incompressibility condition $\boldsymbol{\nabla} \cdot \mathbf{u}=0$, this can also be written as

$$
\frac{\partial u_{x}}{\partial x}+\left[\frac{\partial u_{x}}{\partial y}+\frac{\partial u_{x}}{\partial z}\right]=0
$$

The term within the square brackets is zero by definition because of the choice of the axes. As a consequence $\partial u_{x} / \partial x=0$, so it follows that $\partial C_{x}(t) / \partial t=0$. Thus the initial slope of the LVCF along a streamline is zero. It follows that along the direction of the mean fluid flow the slope is also zero, because the average of the vector tangent to a trajectory is parallel to the vector defining the direction of the flow.

\section{APPENDIX B: COMPUTING STREAMLINES}

A streamline is the trajectory followed by a tracer particle when the system is stationary and the particles have no diffusion. In this section we describe how we computed the stream lines. The problem that has to be solved is simple: given a field $V\left(\mathbf{x}_{i}\right)$ on a lattice $\mathbf{x}_{i}$, construct a flux line $\mathbf{x}(t)$, where $\mathbf{x}$ is a continuous variable. A straightforward procedure to perform this calculation is the Euler method: $\mathbf{x}(t$ $+1)=\mathbf{x}(t)+d t V(\mathbf{x}(t))$. This method is only accurate to first order. We employed the Runge-Kutta method in the midpoint approximation [13], which is accurate to second order in the time step. Higher-order methods were not necessary. The value of $V(\mathbf{x}(t))$ has to be interpolated. In order to compute the off-lattice values of the velocity field, we used a very simple trilinear interpolation, which is the three-dimensional generalization of the linear interpolation $V\left(x_{i}+d x\right)=V\left(x_{i}\right)$ $+d x\left[V\left(x_{i+1}\right)-V\left(x_{i}\right)\right]$.

Although very simple, the approach described above proved to be very robust. We checked that the time step chosen was small enough to ensure a consistent streamline calculation up to the distance used in our simulations. Any possible improvement in the streamline calculation results in a small enhancement of the effect we have pointed out in this paper.
[1] H.C. Brinkman, Appl. Sci. Res., Sect. A 1, 27 (1947).

[2] F.W. Wiegel, Fluid Flow Through Porous Macromolecular Systems (Springer-Verlag, Berlin, 1980).

[3] M. Sahimi, Flow and Transport in Porous Media and Fractured Rock. From Classical Methods to Modern Approaches (VCH, Weinheim, 1995).

[4] A. Acrivos, E.J. Hinch, and D.J. Jeffrey, J. Fluid Mech. 101, 403 (1980).

[5] R.E. Caflisch and J.H.C. Luke, Phys. Fluids 28, 759 (1985).

[6] A.J.C. Ladd, Phys. Rev. Lett. 88, 048301 (2002).

[7] C.P. Lowe and D. Frenkel, Phys. Rev. Lett. 77, 4552 (1996).
[8] D.L. Koch, R.J. Hill, and A.S. Sangani, Phys. Fluids 10, 3035 (1998).

[9] L.J. Dorlofsky and J.F. Brady, Phys. Fluids 30, 3329 (1987).

[10] D.L. Koch and J.F. Brady, J. Fluid Mech. 154, 399 (1984).

[11] A.J.C. Ladd, J. Fluid Mech. 271, 285 (1994).

[12] Y.H. Qian, D. d'Humières, and P. Lallemand, Europhys. Lett. 17, 479 (1992).

[13] W.H. William, S.A. Teukolsky, W.T. Vetterling, and B.P. Flannery, Numerical Recipes in $C$, 2nd ed. (Cambridge University Press, New York, 1993). 